\title{
A Method to Assess Neurological Effectiveness of a Spinal Adjustment for an Individual Patient: A Descriptive Study
}

\author{
John Hart
}

Sherman College of Chiropractic, P.O. Box 1452, Spartanburg, SC 29304, USA

\begin{abstract}
Introduction: A hallmark in health care research is comparison, typically done by comparing groups of patients, e.g., intervention group versus no intervention group. The clinician may be interested in bringing these research methods to the level of the individual patient in practice. Such is done in the present study, where the neurological indicator of resting pulse rate (RPR) is compared pre versus post spinal adjustment, and also compared to instances of no adjustment - for one individual patient. Research indicates that a lower RPR is healthier than a higher RPR.

Methods: Neurological disturbance was operationally defined in the present study as at least two increases in RPR on consecutive visits. Based on this criterion, the patient, over hundreds of RPR measures observed over approximately 2 years, had 16 instances of neurological disturbance; in one of these instances a chiropractic spinal adjustment was given. The 15 other instances were used to estimate a predicted post RPR, which was compared to the observed postadjustment RPR.

Results: Post-adjustment RPR was 67.5 beats per minute (BPM) which was only slightly lower than the average predicted post RPR of 68.1 BPM.

Conclusion: The method described may help clinicians determine if their intervention was neurologically effective. The method also provides normative RPR data for future comparisons of adjustment versus no adjustment. In the present case, the chiropractic adjustment post RPR was better (lower) than the predicted post RPR, but only slightly so.
\end{abstract}

Keywords: Resting pulse rate, biostatistics, chiropractic adjustment.

\section{INTRODUCTION}

\section{Researching the Individual Patient}

The effectiveness of a health care intervention is typically tested at the group level, comparing one group of patients to another group to assess differences between groups. However, such research methods could be applied to the level of the individual patient, which would have an advantage of increased relevancy of the analysis for that patient. In particular, the present study seeks to bring statistical research methods to the level of the individual patient in practice using resting pulse rate to determine whether neurological improvement has occurred following a chiropractic intervention.

\section{Chiropractic Care}

The purpose of chiropractic care is to improve neurological function by adjusting a condition known as vertebral subluxation [1-3]. In chiropractic, this condition essentially consists of a slight vertebral misalignment that results in a neurological disturbance. Adjustment of vertebral subluxation is not intended as a treatment of symptoms or disease, though these may often diminish or resolve following chiropractic spinal adjustment, thanks to the body's innate (inborn) striving

*Address correspondence to this author at the Sherman College of Chiropractic, P.O. Box 1452, Spartanburg, SC 29304, USA;

E-mail: jhart@sherman.edu to maintain and heal itself. The intent of the adjustment is to improve neurological function, to boost general health, similar to the notion that good nutrition and exercise can improve general health.

During care for vertebral subluxation, the chiropractor typically: a) looks for clues as to whether or not the patient's nervous system is improving compared to the previous visit, and b) does not adjust if improvement is noted, even if the improvement is only minimal. Thus, on some chiropractic visits, if the patient's nervous system is considered improving satisfactorily, no adjustment is given, even if symptoms are present.

\section{Resting Pulse Rate}

An option for assessing neurological function in subluxation-centered chiropractic is resting pulse rate (RPR). RPR: a) is a clinical neurological assessment; [4-5] b) is supported by outcomes research showing that people with lower RPR tend to be healthier (e.g., live longer) than those with higher RPR; [6-7] c) has good agreement with resting heart rate derived from ECG [8] as well as good agreement with heart rate variability; [9] and d) may decrease (improve) following chiropractic adjustment [10-12].

\section{Purpose of the Study}

The present study describes a method to calculate normative RPR data for the individual patient, where no 
chiropractic adjustment was given. The method then compares an instance where adjustment was given to an instance (or prediction) where no adjustment was given.

\section{METHODS}

\section{The Patient}

A 59 year old male chiropractic wellness patient signed a consent form for this study, which was approved by the Institutional Review Board at Sherman College of Chiropractic. The patient received training on how to take his RPR and self-measured the RPR over an approximate 2 year period, from May 2014 to March 2016 for a total of 328 measurements. Patient symptoms were not a focus in the study because: a) the patient did not have any that were of consequence, and b) the focus of the study was neurological function rather than symptomatic sequelae.

\section{Measurements}

The RPR measurements were obtained: a) With the patient in the seated position after a minimum of 5 minutes seated rest; b) at the radial artery, counting for 30 seconds, two trials, with a 30 second interval between trials. The average of the two measurements was multiplied by 2 to achieve a beat per minute (BPM) value and was used for analysis; c) counting the first beat as 1 (instead of 0 ); d) using a digital timer, e) during the same hour of day (10:00 AM - 11:00 AM); f) at least two hours after food intake; and g) at least 12 hours after alcohol consumption. During the study period the patient did not take any medication, did not smoke, and does not recall having drunk any coffee during the study period (he rarely drinks coffee).

\section{Operational Definition of Neurological Disturbance}

In this study, the operational definition of a neurological disturbance consisted of at least two increases in RPR on consecutive measurement days (now referred to as "visits"). This theoretical definition is based on long term studies showing that an increase in RPR over time is associated with worse health outcomes (e.g., higher death rates) compared to no increase or decreased RPR over time. In the present study, 16 instances of neurological disturbance were identified. In one of the instances, the patient consulted a chiropractor for the neurological disturbance. The chiropractor adjusted the patient using a percussion instrument for atlas subluxation. In the other 15 instances, adjustment was not given, since the patient did not seek chiropractic care for these instances. These instances (where no adjustment was given) allowed for a retrospective study of the natural history of RPR variation, without intervention. This further allowed for a comparison of adjustment versus no adjustment with the patient serving as his own control. On some of the instances of neurological disturbance there were two increases on consecutive visits while in other instances there were three increases on consecutive visits.

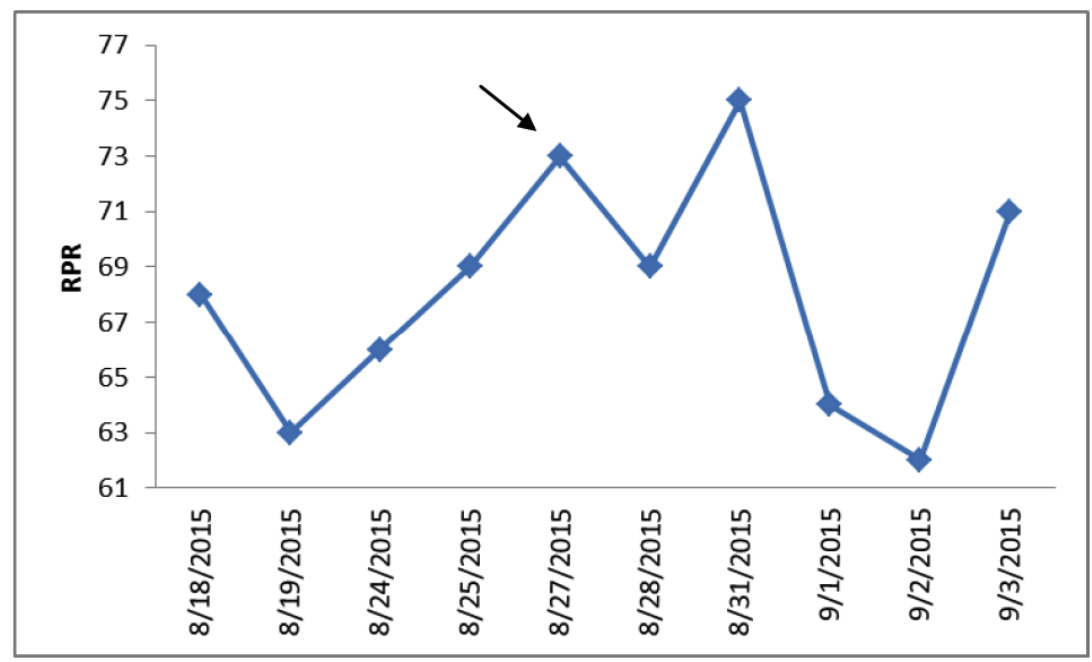

Figure 1: Pre and post resting pulse rates in beats per minute for the instance of adjustment (arrow). In this instance there were three increasing pre RPRs. The 8-27-15 RPR measurement is the last pre in this instance and adjustment was given immediately following this measurement. If this was an instance of no adjustment, the beginning of the "post" period would be marked by the first RPR to not increase following the last two increasing RPRs, which would be 8-28-15. General improvement (decrease) following the adjustment was considered to have occurred through and including 9-2-15, six days following the last pre and represents the post period. The post is the average of RPRs in the post period Other RPR measurements (8-18-15, 819-15, and 9-3-15) are provided to help further illustrate the selection method for pre and post. 
Table 1: Pre and Post Resting Pulse Rate in Beats Per Minute in all Instances of Neurological Disturbance*

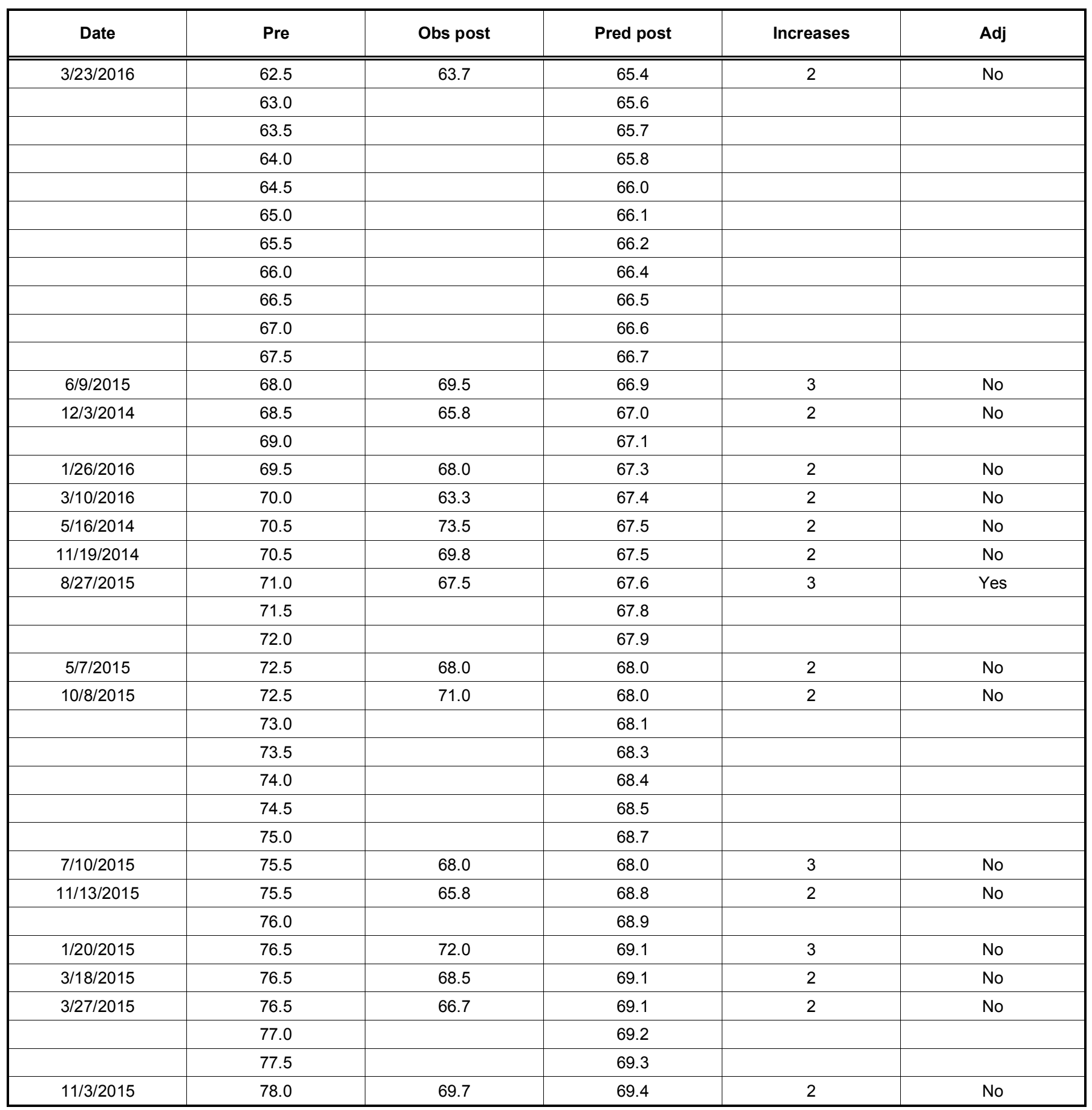

*Sorted by Pre, from lowest-to-highest and includes the one instance of adjustment (on 8-27-15). Date = date of last increased pre RPR measurement. Pre is the mean of two increased RPR readings on consecutive measurement sessions. Post is the mean of the next RPRs over a 6 day period from the second pre. Obs post $=$ observed post. Pred post $=$ predicted post. Blanks in the various columns are where there were no observed pre RPR values. For these blanks, post RPR was predicted from predicted pre RPR. Increases = number of consecutive increases in the observed pre.

In each instance of neurological disturbance, the mean of the last two increased RPRs (referred to now as "pre") were compared to the mean of the next RPRs over a 6 day period which began on the day of the last pre. The first post RPR was the first one to not increase following the 2 or 3 consecutive increasing RPRs (Figure 1). The mean RPR during this 6 day period is now referred to as the "post." A criterion for inclusion as a post period was that there had to be at least two RPR measurements in the 6 day post period. It will be noted that the terms "pre" and "post" are used not only in the instance of adjustment but also in instances of no adjustment. The amount of time in the post period, 6 days from the last increasing pre, was selected 
because it was the amount of time where improvement (decrease) in RPR occurred following the one adjustment in the study (Figure 1). Thus, it was desirable that the comparison times in adjustment versus no adjustment be the same, so that an "applesto-apples" comparison could be made.

\section{Analysis}

The dependent (response) variable of the study was post RPR while the independent (predictor) variable was pre RPR. The observed RPR values were calculated with one decimal place precision, ranging from 62.5 BPM to 78.0 BPM. Some RPR values were not observed in the study within this range. To fill in the gaps, predicted average post RPR values were calculated from predicted pre values (Table 1). The prediction of post RPR (for observed and predicted pre RPR values) was based on the 15 instances of no adjustment using two steps in Stata IC 12.1 (Stata Corp., College Station, TX): a) linear regression and then b) the "predict y" procedure, y being the response variable (= post RPR). This prediction can also be done in Excel. [13]. In this way, predicted post RPR for corresponding (but missing) pre RPR is based on observed (actual) RPR values. Thus, the data consisted of observed (actual pre and post) and predicted (predicted pre and post) RPR data.

A sub-analysis was performed according to number of consecutive increases in the pre (the two or three consecutive increases previously described). This was done in case there was some effect from the number of consecutive increases (e.g., three RPR increases perhaps followed by higher post RPR compared to two increases in the pre). Among the 15 instances of neurological disturbance without adjustment, 12 consisted of two pre increases on consecutive visits (where pre RPR continued to range from 62.5 BPM to 78.0 BPM). The remaining three instances consisted of three pre increases on consecutive visits (where pre RPR ranged from 68.0 BPM to 76.5 BPM). Changes in these ranges were accounted for in the sub-analysis. The main analysis included all data (two and three consecutive increases).

An additional check on the quality of the data was to assess repeatability of the patient's two RPR measurements (taken within a 90 second time frame, as described above). Here, the intra-class correlation coefficient (ICC) was calculated, also in Stata. ICC is a suggested test for assessing repeatability of continuous data measures such as RPR [14].

\section{Research Hypothesis}

The research hypothesis of the study is that post RPR in adjustment is expected to be better (lower) than post RPR without adjustment and/or predicted post RPR in the event there is no matching observed pre RPR.

\section{RESULTS}

\section{No Adjustment}

For relatively low pre RPRs, predicted post RPRs were higher than corresponding pre RPRs (Table 1, Figure 2). As pre RPR increased, predicted post RPR also increased, and the two values became equal at the pre value of 66.5 BPM (Table 1). After this, predicted post RPR became smaller than corresponding pre RPR values (Table 1).

The mean pre RPR for the 15 instances of no adjustment was $72.2 \mathrm{BPM}$, standard deviation (SD) = 4.3 , compared to $68.0 \mathrm{BPM}(\mathrm{SD}=2.9)$ for post RPR (also without adjustment, Table 2).

\section{Adjustment versus No Adjustment}

Observed pre RPR in the one instance of adjustment was 71.0 BPM, followed by an observed post RPR that decreased (improved) to 67.5 BPM. Since there were no matching observed pre RPR values (of $71.0 \mathrm{BPM}$ ) without adjustment, a predicted RPR post was used for comparison. The predicted post RPR value here (where no adjustment was given for a pre RPR of 71.0 BPM) was: a) 67.6 BPM when all instances were analyzed (Table 1, Figure 3), b) 67.2 BPM when analysis was limited to two consecutive increases for pre RPR, and c) 69.6 BPM when analysis was limited to three consecutive increases for pre RPR. Thus, two out of the three analyses here showed that the adjustment was followed by a better (lower) post RPR compared to the predicted post RPR.

\section{Reliability of The Data}

The patient's repeatability of the RPR measurements was acceptable, with an ICC value of $0.863(p<$ 0.0001 ). The absolute difference between the two RPR measurements ranged from 0 to $12 \mathrm{BPM}$, with the breakdown of differences as follows: $123(37.5 \%)=0$ BPM, $164(50 \%)=2$ BPM, $36(11.0 \%)=4$ BPM, $4(1 \%)$ $=6 \mathrm{BPM}$, and $1(0.3 \%)=12 \mathrm{BPM}$. The one instance for which the absolute difference was 12 BPM did not happen to be a part of any pre or post analyses, and was therefore only used here in reliability analysis. 


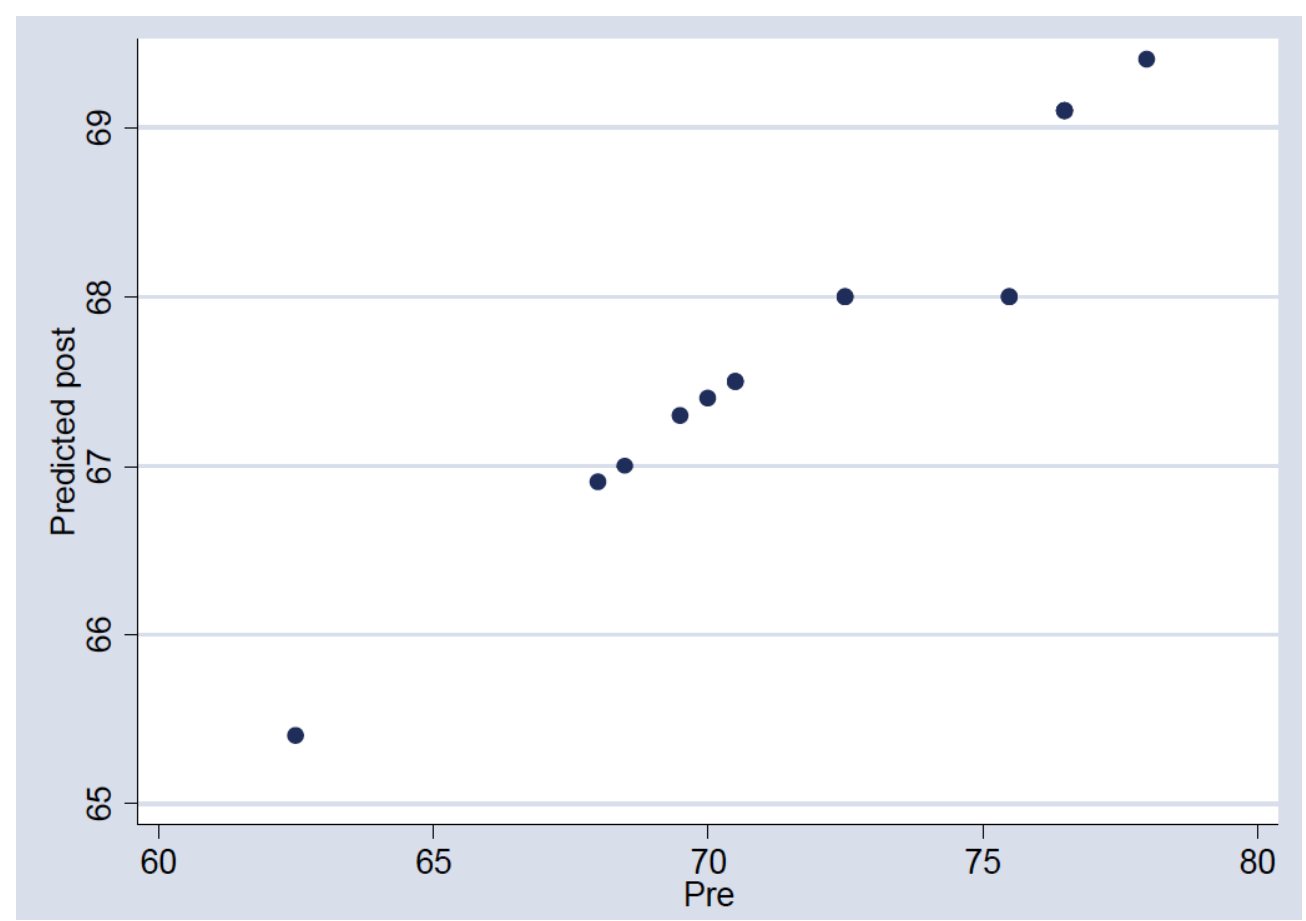

Figure 2: Scatter plot of predicted post RPR versus pre RPR in instances of no adjustment. Lower pre correspond to predicted posts that are larger than the pre (e.g., the first data point on the lower left represents a pre of 62.5 BPM and its predicted post is 65.4 BPM, as shown in Table 1). Higher pre correspond to predicted posts that are smaller than the pre (e.g., the last data point on the upper right represents a pre of 78.0 BPM and its predicted post is 69.4 BPM, as shown in Table 1).

Table 2: Descriptive Statistics for Observed Resting Pulse Rates in Instances of No Adjustment

\begin{tabular}{|c|c|c|c|c|c|}
\hline Type & $\mathbf{n}$ & Mean & SD & Minimal & Maximum \\
\hline \hline Pre & 15 & 72.2 & 4.3 & 62.5 & 78.0 \\
\hline Post & 15 & 68.0 & 2.9 & 63.3 & 73.5 \\
\hline
\end{tabular}

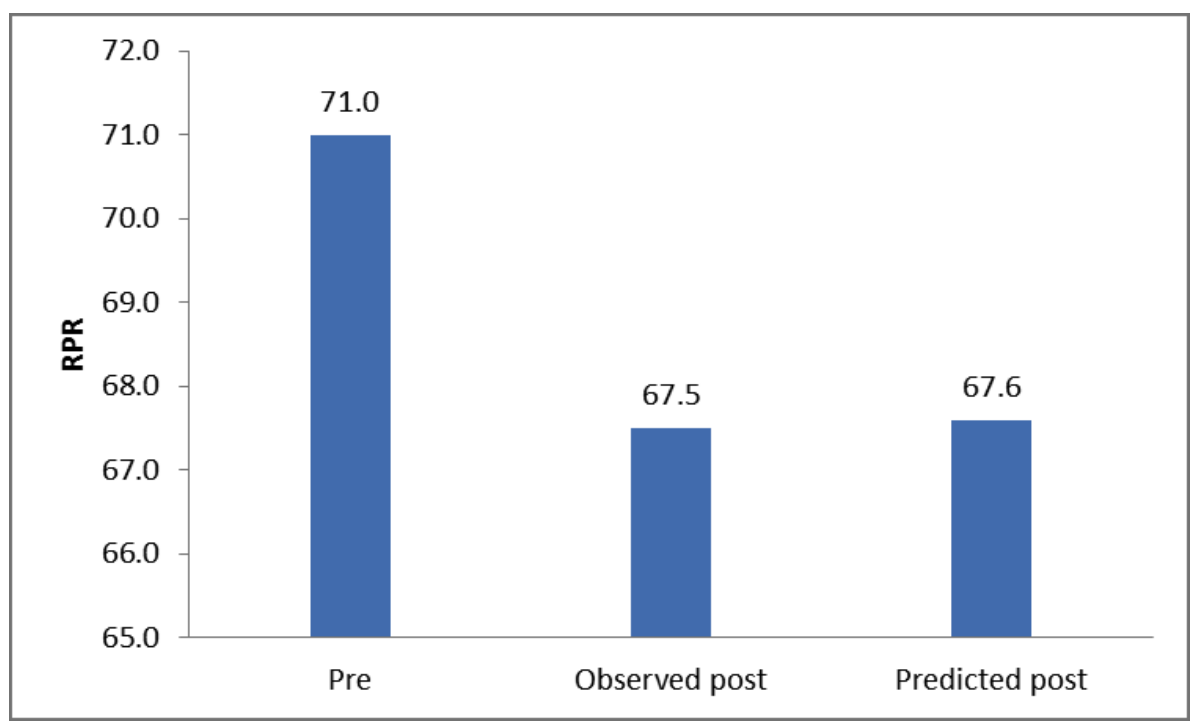

Figure 3: Adjustment versus no adjustment. Pre is mean of last two increasing resting pulse measurements prior to the spinal adjustment on 8-27-15. Observed post is the mean of the four RPRs following the adjustment, to 9-2-15, as shown in Figure 1. Predicted post is based on all 15 instances of no adjustment (as explained in Methods). Improvement in observed post is only slightly better (lower) than the predicted and therefore may not be clinically significant. Nonetheless, having a chart like this at least allows the clinician to be informed in regard to the comparison. 
Table 3: Lowest and Highest Pre RPR in the Study*

\begin{tabular}{|c|c|c|}
\hline Pre RPR & Predicted Post RPR & Post - Pre difference \\
\hline \hline 62.5 & 65.4 & 2.9 increase \\
\hline 78.0 & 69.4 & 8.6 decrease \\
\hline
\end{tabular}

*This is one example where post-pre difference may not be an appropriate measure to compare instances of neurological disturbance (as explained in the Discussion section). Both examples are from instances of no adjustment.

\section{DISCUSSION}

The main purpose of the study was to describe a method of investigation that could be applied to the individual patient. In particular, the method assesses the possible neurological effectiveness of a single chiropractic adjustment by comparing post RPR in adjustment versus no adjustment (or predicted post RPR). In the present case, the observed post RPR in adjustment was lower than its average predicted post RPR. This suggests that there was greater neurological improvement following the adjustment compared to no adjustment when matching pre RPRs are used.

\section{Limitations to the Study}

The following limitations apply: a) An RPR value in the study is a single value (based on the mean of multiple RPR values; two values for pre and at least two for post), without a corresponding range or confidence limit with which to convey uncertainty around the single value. A similar situation exists in regard to other clinical data such as systolic blood pressure, where a single numerical value is used, e.g., $140 \mathrm{~mm} \mathrm{Hg}$, where people are urged to have a reading below this number, and where a range or confidence limit is often not included; [15] b) there was only a 0.1 BPM difference between the observed and predicted post RPRs in adjustment. This difference may or may not be clinically significant. In a longer term study on patients with hypertension, a 1 BPM change was associated with a $1 \%$ change in mortality risk [6]. Extrapolating from that study, it may be that a 0.1 BPM change may represent a $0.1 \%$ change in mortality risk; c) there were only three observations in the sub-analysis of three consecutive increases in RPR; and d) the patient selfreferred in the instance of the one adjustment. Thus, the intervention was not random among the 16 instances of neurological disturbance, thereby limiting generalization to instances of no adjustment.

\section{Up and Down Variability}

As previously mentioned, lower pre RPR tended to be followed by predicted post RPR that are higher than the corresponding pre RPR; and higher pre RPR tended to be followed by lower corresponding predicted post RPR. This is consistent with the cliché, what goes up, tends to come down. The author would like to add to this cliché that for RPR, what goes down, tends to go up. The up-and-down variability of RPR is why prepost differences should not be the metric used for comparing instances (e.g., between adjustment versus no adjustment) when pre RPR values are different between instances. An example is provided in Table 3 showing the lowest and highest pre RPRs in the study without adjustment (62.5 BPM and 78.0 BPM respectively). If pre-post differences were used as the comparison metric, it may appear that greater neurological improvement followed the pre 78.0 BPM finding compared to the pre 62.5 BPM. However, natural variability indicates that what is down (the pre 62.5 BPM) tends to go up (to the post 65.4 BPM), and what is up (the pre 78.0 BPM) tends to go down (to the post of 69.4 BPM).

The recommended method is to compare post RPR across instances which have the same or even similar (not exactly the same) pre RPR. Taking the average of multiple observed post RPR (to calculate a predicted post RPR) accounts for measurement variability. Using measurements that fall near to the observed pre RPR avoids the problem of predicting outside the range of the data, and also avoids problems with non-linearity. In instances where matching (or similar) observed pre values are not available in instances of no adjustment (to compare to adjustment), predicted post RPR based on all the other data in instances of no adjustment would also be acceptable.

\section{Self-Maintenance by the Body}

A higher pre RPR that is followed by a lower corresponding post RPR, without adjustment (e.g., the pre 78.0 BPM followed by an observed post RPR of 69.7 BPM and predicted post of 69.4 BPM), appears to be an example of the body's innate (inborn) striving to maintain and heal itself, even without intervention. This striving is a principle that is well-recognized by subluxation-centered chiropractors and is a reason why the number of adjustments given is kept to a bare minimum, allowing the body to do most of the healing (improving nervous system function in this case) itself. 


\section{Clinical Usefulness}

The various pre RPR values in Table 1 in instances of no adjustment (observed and predicted) are useful for future comparison of other instances of adjustment versus no adjustment (with matching pre RPRs). For example, a future adjustment that is given with a pre RPR value of 65.0 BPM would be expected to show a post RPR value less than the predicted post RPR of 66.1 BPM (Table 1).

In instances of no adjustment, an observed (actual) RPR data point would seem to carry more weight (reliability) than a predicted value, if there are multiple observed values where the corresponding post values can be averaged (to account for variability). If, however, there is only one available observed value, then a predicted post, based on all the data in instances of no adjustment would seem to be more reliable. Moreover, multiple observed instances of the same pre RPR, resulting in averaging of the corresponding post RPRs, would further increase the reliability of that observed post RPR average.

\section{Next Steps}

Future study could include: a) different time frames and number of RPR measurements in the pre and post periods; b) frequent update of the normative data where new instances of neurological disturbance are observed in instances of no adjustment; c) applying the method in other chiropractic patients receiving care for vertebral subluxation, and with other chiropractic techniques; and d) validating the method with an outcomes-based research design.

\section{CONCLUSION}

The present study compares the neurological assessment of resting pulse rate (RPR) in an instance of adjustment versus no adjustment. The method may be of interest to practicing clinicians who have a neurological focus (e.g., chiropractors in subluxationcentered chiropractic). The method also calculated various pre RPR values for future comparison of other adjustments with matching pre RPR, where the patient is his or her own control.

\section{REFERENCES}

[1] Palmer DD. Text-Book on the Science, Art, and Philosophy of Chiropractic. Portland, OR: Portland Printing House 1910.

[2] Association of Chiropractic Colleges [Internet page]. Chiropractic paradigm. The subluxation. Accessed on December 16, 2015 from: http://www.chirocolleges.org/ paradigm_scope_practice.html

[3] Sherman College of Chiropractic [Internet page]. Sherman College focuses on vertebral subluxation. Accessed on January 29, 2016 from: http://www.sherman.edu/? page_id $=2335$

[4] Mensink GBM, Hoffmeister $H$. The relationship between resting heart rate and all-cause, cardiovascular and cancer mortality. Eur Heart J 1997; 18: 1404-1410. http://dx.doi.org/10.1093/oxfordjournals.eurheartj.a015465

[5] Hsia J, Larson JC, Ockene JK, Sarto GE, Allison MA, Hendrix SL, Robinson JG, LaCroix AZ, Manson JE. Resting heart rate as a low tech predictor of coronary events in women: prospective cohort study. Brit Med J 2009; 338: 577580.

http://dx.doi.org/10.1136/bmj.b219

[6] Paul L, Hastie CE, Li WS, Harrow C, Muir S, Connell J, Dominiczak AF, Mclnnes GT, Padmanabhan S. Resting heart rate pattern during follow-up and mortality in hypertensive patients. Hypertension 2010; 55(part 2): 567 574.

[7] Jouven X, Empana JP, Escolano S, Buyck JF, Tafflet M, Desnos M, Ducimetiere P. Relation of heart rate at rest and long term ( $>20$ years) death rate in initially healthy middle aged men. Am J Cardiol 2009; 103: 279-283. http://dx.doi.org/10.1016/j.amjcard.2008.08.071

[8] Erikssen J, Rodahl K. Resting heart rate in apparently healthy middle-aged men. Eur J Appl Physiol O 1979; 42(1): 61-69. http://dx.doi.org/10.1007/BF00421105

[9] Hart J. Association between heart rate variability and manual pulse rate. J Can Chiropr Assoc 2013; 57(3): 243-250.

[10] Hart J. Reduction of resting pulse rate following chiropractic adjustment of atlas subluxation. Ann Vert Sublux Res 2014 March 3: 16-21.

[11] Zhang J, Dean D, Nosco D, Strathopulos D, Floros M. Effect of chiropractic care on heart rate variability and pain in a multisite clinical study. J Manip Physiol Ther 2006; 29: 267274.

http://dx.doi.org/10.1016/j.jmpt.2006.03.010

[12] Hart J. Comparison of resting pulse rates in chiropractic students versus the general population. Top Integr Health Care 2012; 3(4): ID 3.4005.

[13] Zaiontz C [Internet page]. Real statistics using Excel. Accessed on April 2, 2016 from: http://www.realstatistics.com/regression/confidence-and-prediction-intervals/

[14] Acock A. A gentle introduction to Stata. $3^{\text {rd }}$ Edition. College Station, TX: Stata Corp 2010

[15] American Heart Association [Internet page]. Study backs guidelines: People with hypertension should get top number below 140. Accessed on April 3, 2016 from: http://news.heart.org/study-backs-guidelines-people-withhypertension-should-get-top-number-below-140/ (http://creativecommons.org/licenses/by-nc/3.0/) which permits unrestricted, non-commercial use, distribution and reproduction in any medium, provided the work is properly cited. 